\title{
DBN theory in constructing multi-sensor FDIR model
}

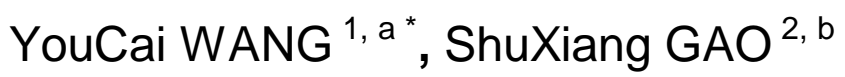 \\ ${ }^{1}$ High-tech Institute, Fan Gong-ting South Street on the $12^{\text {th }}$, Qing Zhou, Shan Dong, China \\ awangyoycai@126.com
}

Keywords: Multi-Sensor, DBN, FDIR, Global Optimization.

Abstract. In this paper, the DBN probabilistic network model is used to realize the fusion of multi sensor uncertainty information, and using the Active DBN detection method to research the global FDIR implementation strategy and optimization methods of fusion order and partial fusion. An improved hierarchical multi-level FDIR fusion model and algorithm is proposed, and the fault-tolerant principle and performance of the FDIR mode are analyzed. The result of simulation shows that the new optimizing method has high accuracy, reliability and fault tolerance.

\section{Introduction}

As an optimal state estimator, The Kalman filter algorithm is easy to implement in real time optimal recursive filtering on the computer. Therefore, information fusion technology based on Kalman filter is adapted to the Fault Diagnosis of multi-sensor information systems. But in the background of complex applications for multi-sensor information system, the large-capacity, diversity and complexity information improve the comprehensive perception of the state of the system capacity, and also introduce a number of risks and uncertainties to the system.

In practical applications, the uncertainties which contains in Sensors information reduce the reliability of detection systems that make the fault detection and diagnosis for sensor system very difficult, it needs to measure the uncertainty of multi-sensor system. As a directional chain structure model [1] DBNs reflects the causality of random variables and time series data by constructing a probabilistic model, it can provide a means for dealing with the uncertainty of multi-sensor information.

\section{The multi-sensor fault detection method based on DBN}

The Bayesian networks (BNs) can model the correlation and uncertainty of events, so it is suitable for multi-sensor detection, but not modeling dynamic events. As a generalization of the Kalman filter model (KFMs) and Hidden Markov Models (HMMs), DBNs provides a good way to represent the state space model [2].

Consider a discrete random process, whenever a new measurement (data set) is obtained, the subscript $t$ increased by 1 . The joint probability distribution of the previous time $(t=1)$ to the next moment $(t=T)$ can be expressed by the following equation.

$$
P\left(X_{1: T}\right)=\prod_{t=1}^{T} \prod_{i=1}^{n} P\left(X_{i}^{t} \mid \pi\left(X_{i}^{t}\right)\right)
$$

In Eq. 1, $X_{i}^{t}$ represents node $i$ at time $t, \pi\left(X_{i}^{t}\right)$ represents the results event of $X_{i}$ at time $t$. The DBN is used to analyze and process the general time series data, sensor family as random variables is incorporated into the Bayesian network structure to form a coherent fault detection structure. In order to show the uncertainty of the sensor, a layer of information distribution variable $\left\{I_{1}, \ldots I_{m}\right\}$ is added to connect sensors and intermediate variables in the DBN structure. So a coherent detection structure can be constructed as shown in Fig. 1. As the only observable variable sensor family is at the bottom level of the link, and the evidence information is obtained by sensor. 


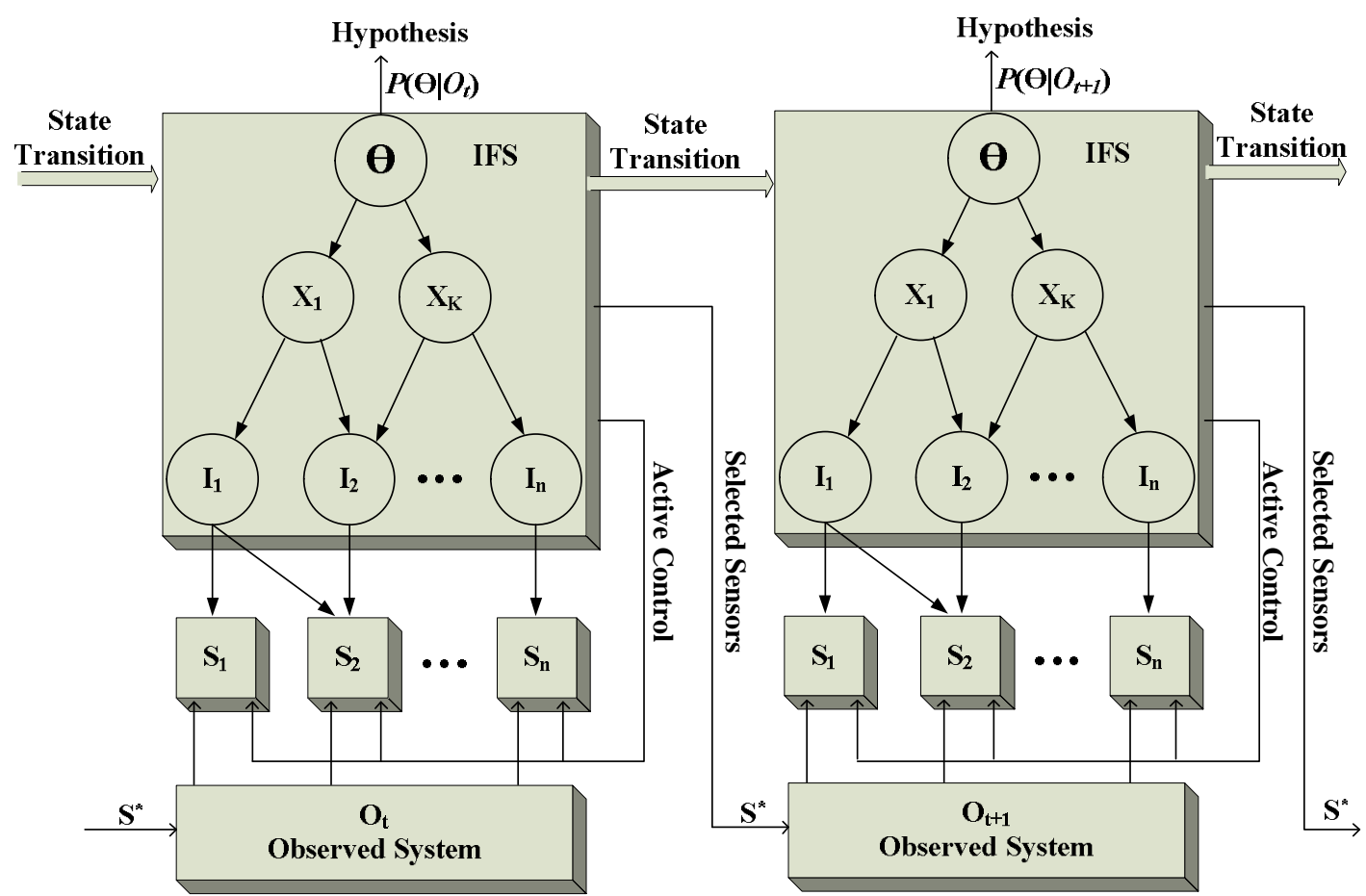

Fig. 1 Hierarchical distribution of information variables and Sensor selection

In order to deal with the uncertainty of the sensor in the detection structure with the probability network, adding an additional variable layer called the information distribution layer between the sensor and the intermediate parameter. The uncertainty error of the sensor measurement is quantified by the value of the information distribution variable and the conditional probability of the sensor. So the uncertainty of sensor measurement is integrated into the detection system that update the probability description of the hypothetical data.

\section{The Active DBN detection method}

The purpose of active fault detection is to select the information which is the most closely related to decision making. In order to achieve the overall efficiency, and select the most related sensor data sets to describe the current target. Its mathematical description is as follows, assuming there are $m$ available sensors $S i, i=1, \ldots, m$, and use them to measure the environmental information. Let $\Theta$ represent a series of probability hypothesis $\theta_{k}$, among them $k=1, \ldots K$. Make sensors $S=\left\{S_{1}, \ldots S_{n}\right\}$ represents the selected set of sensors at the $t$ time, $n \in\{1, \ldots, m\}$. The measurement data of the sensor $S i$ at the $t$ time can be expressed as $O_{t}\left(S_{i}\right)$, and $O_{t}\left(S_{i}\right)$ of the $i$-th sensor which belongs to a known finite state set consisting of $e_{1}^{(i)}, \ldots e_{L}^{(i)}$. Make $O_{t}=\left\{o_{t}\left(S_{1}\right), \ldots, o_{t}\left(S_{n}\right)\right\}$ express the available information of the current time $t$, and the sensor selection at $t+1$ time is based on the $O_{t}$. The Active fault detection can be divided into four stages every moment.

(1) Sensor selection: After receiving $O_{t}$, select the optimal sensor set for the next time $t+1$ based on the system state;

(2) Observation: Observed results $O_{t+1}\left(S_{i}\right), i=1, \ldots, n$, get the new sensor information $O_{t+1}$, $S_{i} \in S^{*}$

(3) Condition assessment: Using DBN inference algorithm to calculate the posterior probability $\mathrm{p}\left(\Theta_{t+1} \mid O_{t+1}\right)$;

(4) Make decisions: If the reliability of the current scheme (reliability) is high enough, make a decision; otherwise, end process and re-start sensor selection for further observation.

The Active control is to choose the available sensor for the next time by considering only the possible output of the sensor, the selected sensors have the ability to anticipate and minimize the 
uncertainty of the information. In the $t$ time, the observing system calls the selected sensor and generates the sensor information $O_{t}$ which is the true output of the selected sensor combination.

Generally, the efficacy function is composed of two parts: the information gain $u_{l}$ and the Information-Loss $C(S)$, it can be used to drive the sensor $S$. Use $u_{2}=1-C(S)$ to convert loss to cost savings that make $u_{1}$ and $u_{2}$ both represent gain, Because of $u_{1}$ and $u_{2}$ are functionally independent of each other [3], so the Multiple linear efficiency function can be obtained as follows.

$$
U\left(u_{1}, u_{2}\right)=\left(k_{1} u_{1}+1\right)\left(k_{2} u_{2}+1\right)
$$

In Eq. $2, k_{1}$ and $k_{2}$ are the preferred parameters, and $k_{1}+k_{2}=1 . u_{1}$ and $u_{2}$ need to be normalized to match the parameters.

According to the Information theory, the interactive information specific measures the average gain of the sensor information before and after the selected time. As shown in Fig.1, start with the information entropy expectation [4], assuming $\Theta$ is related to the all possible output of the sensor $S_{i}$. Under the given $S i$, the information entropy expectation measures the degree of uncertainty in $t$ (Omit the subscripts $t$ for making equation clearly). Its expression is as follows.

$$
H\left(\Theta \mid S_{i}\right)=-\sum_{S_{i}} \sum_{\Theta} P\left(\theta, s_{i}\right) \log P\left(\theta \mid s_{i}\right)
$$

In Eq. 3, $s_{i}$ indicates the value of the sensor $S_{i .}$ And under the premise of $S_{i}$ can be obtained, subtract $H\left(\Theta \mid S_{i}\right)$ from the initial uncertainty of $\Theta$ before obtain $S_{i}$ and $H\left(\Theta \mid S_{i}\right)$ Specifically, The quantity of information about $\Theta$ can be obtained by the following equation.

$$
\begin{aligned}
I\left(\Theta ; S_{i}\right) & =H(\Theta)-H\left(\Theta \mid S_{i}\right) \\
& =-\sum_{\Theta} P(\theta) \log P(\theta)+\sum_{S_{i}}\left\{P\left(S_{i}\right) \sum_{\Theta} P\left(\theta \mid S_{i}\right) \log P\left(\theta \mid S_{i}\right)\right\} \\
& =\sum_{\Theta} \sum_{S_{i}} P\left(\theta, S_{i}\right) \log \frac{P\left(\theta \mid S_{i}\right)}{P(\theta)}
\end{aligned}
$$

$I\left(\Theta ; S_{i}\right)$ quantifies the ability of $\Theta$ to reduce the degree of uncertainty of information, so the public information $I\left(\Theta ; S_{i}\right)$ of sensor family $S=\left\{S_{1}, \ldots S_{n}\right\}$ can be expressed as follows.

$$
\begin{aligned}
I(\Theta ; S) & =H(\Theta)-H(\Theta \mid S) \\
& =\sum_{\Theta} \sum_{S 1 \ldots S_{n}}\left\{P\left(\theta, S_{1}, \ldots S_{n}\right) \log \frac{P\left(\theta \mid S_{1}, \ldots S_{n}\right)}{P(\theta)}\right\}
\end{aligned}
$$

In the $t$ time, the values of $P\left(\theta, s_{1}, \ldots s_{n}\right)$ and $P\left(\theta \mid s_{1}, \ldots s_{n}\right)$ can be obtained by using DBN inference algorithm and considering the sensor observations at the $t-1$ time and the current $t$ time. Eq. 5 provides criteria for determining the ability to reduce uncertainty when the Sensor combination has been given.

To make $S$ as a space for all sensors or sensor groups, $S^{*}$ is an optimized sensor combination. Using the proposed theoretical framework, the following sequential decision-making process can be obtained.

$\%----$ Sequential Decision-Making Process -----\%

$1 t \leftarrow 0$

$2 S^{*}=\arg \max _{S \in S} U(I(\Theta ; S), C(S))$

3 while $t<T$

4 Activate all $S_{i} \in S$, and get $O_{t}$

5 Use DBN to reference and get $P\left(\Theta \mid O_{t}\right)$

6 If there is sufficient certainty, make decisions

7 else $S^{*}=\arg \max _{S \in S} U(I(\Theta ; S), C(S))$

$8 t \leftarrow t+1$

$$
\%
$$

As the detection structure DBN provides a probabilistic analysis theory framework with sequential hierarchical for the detection system, and it can also describe and deal with the uncertainty and the 
instantaneous variations of the sensor data, It reflects the timing characteristics of decision-making. But the active control and management of the testing process need to design effective, real-time sensor selection method, and the method should allow the detection system to independently select and call the most closely decision-related sensor combination.

\section{The implementation strategy of global FDIR}

The Improved Kalman filter [5] can provide the fault detection information to the global filter, so that the multi sensor system has the ability of global FDIR (Fault Detection, Isolation and Recovery).In practice, the slowly changing faults can be detected only when the fault information is accumulated to a certain degree. That will contaminate the global estimation information, so need to improve the strategies and methods for multi-sensor hierarchical / multi-level / variable structure FDIR.

Aiming at the hysteresis of soft fault, The Hierarchic Alliance of Filter transforms the two-level structure of the federated filter into a multi-level structure [6]. In the federated filter structure, assuming the global filter is $G$, its global state estimation and covariance is $\hat{X}_{g}$ and $P_{g}$ respectively, $G^{i}$ is a backup of the federal filter for the estimation of the $i$-th local filter, make $\hat{X}_{g^{i}}$ and $P_{g^{i}}$ respectively represent the global estimation and covariance of $G^{i}$, according to the federal filter detection algorithm is derived as follows.

$$
\begin{aligned}
& \hat{X}_{g^{i}}=P_{g^{i}}\left(P_{m}^{-1} \hat{X}_{m}+\sum_{\substack{j=1 \\
j \neq i}}^{n} P_{j}^{-1} \hat{X}_{j}\right) \\
& P_{g^{i}}^{-1}=P_{m}^{-1}+\sum_{\substack{j=1 \\
j \neq i}}^{n} P_{j}^{-1}
\end{aligned}
$$

$P_{m}$ and $\hat{X}_{m}$ are respectively the estimation error covariance and the state estimation of the main filter in the backup filter. Similarly, $G^{i j}$ is defined as a backup filter that removes the $i$-th and the $j$-th local estimation whose global estimation and covariance are $\hat{X}_{g^{i j}}$ and $P_{g^{i j}}$. And so on, we can continue to define the backup filter $G^{i j k \cdots}$. Make $G^{i}$ as the First-generation Backup Filter and $G^{i j}$ as the Second-generation Backup Filter and so on which can form the Dendriform Hierarchic Alliance structure. In the Hierarchical federal structure, each filter can be more easily derived from its upper primary filter (the parent filter).

For a filter family which has a common parent filter, the FDIR strategy of the global detection system based on filter family can be described as follows. If the sensor fault meets the following conditions:

(1) At most only one sensor fault occurs in the detectable time period;

(2) The jump variant fault is not a frequent occurrence. Otherwise reset, system is not available.

The FDIR of the global detection system can be carried out by using the hierarchical federated filtering structure:

a. There is only one filter family run when the system is running;

b. As the optimal filter, father filter gives real time estimates online;

c. Other filters as a backup filter use a backup filter to replace the parent filter when the local filter failure has been detected by the subsystem fault diagnosis method;

d. Replace the parent filter containing the fault information with the backup filter, and make it to be parent filter. Correspondingly, the new filter family is generated as a backup.

The multi-sensor global optimization model can be formed [7] for practical applications, by the combination of the partial information optimization detection model and the global hierarchical multilevel FDIR detection algorithm. 


\section{Mathematical Simulation and Analysis}

Take a combination of SINS/GPS, SINS/RDSS, SINS/Doppler, SINS/CNS navigation sensor as an example, the simulation data are as follows.

The simulation initial conditions are the same for each local system: the initial attitude angle error $\Delta \theta(0)=\Delta \psi(0)=\Delta \gamma(0)=20^{\prime}$, the initial velocity error $\Delta V_{x 0}=\Delta V_{y 0}=10 \mathrm{~m} / \mathrm{s}$, the initial position error $\Delta L_{0}=\Delta \lambda_{0}=4^{\prime}$. The constant drift of INS gyro is $0.5^{\circ} / \mathrm{hr}$, the gyro random drift $\sigma=0.5^{\circ} / \mathrm{hr}$, the Accelerometer constant error is $10^{-4} \mathrm{~g}$, the Speed meter random noise $\sigma=10^{-4} \mathrm{~g}$. The research object moves along the designed simulation trajectory, the simulation time is 1000s. The random pseudo-range measurement error of GPS is $20 \mathrm{~m}$, the random Delta pseudo-range measurement error is $0.05 \mathrm{~m} / \mathrm{s}$, and the correlation time for the range rate error of GPS is $300 \mathrm{~s}$.

Fault information setting: 500 510s, Amplitude: the pseudo-range is 50m, the Delta pseudo-range is $1 \mathrm{~m} / \mathrm{s}$; System noise is set to colored noise. According to the relevant analysis, other sensor system parameters and measurement errors are selected in general experience of the sensor.

(1) The accuracy Of Double-Star point positioning is $100 \mathrm{~m}$, Difference is $20 \mathrm{~m}$, and takes $100 \mathrm{~m}$ in simulation;

(2) Both the Horizontal dip $\alpha$ and the horizontal angle $\beta$ are $45^{\circ}$, and the error of fixed angles $\delta \alpha=\delta \beta=0.001^{\circ}$, the radar frequency $\lambda=9500 \mathrm{kHz}$, the Doppler shift measurement error $\delta f=6.3 \times 10^{-5} \mathrm{~Hz}$, and the correlation time for velocity measurement random error is $300 \mathrm{~s}$.

(3) Navigation satellite data of CNS from the Sky2000 Master Star catalogue, the star sensors Installation error is $5^{\prime}$, the random error $\sigma=\sqrt{3}^{\prime}$, the measurement error is $20^{\prime}$.

As shown in the following figure, the simulation curve shows the detection estimation accuracy and the performance of the fault detection based on the FDIR global optimization detection model and algorithm.

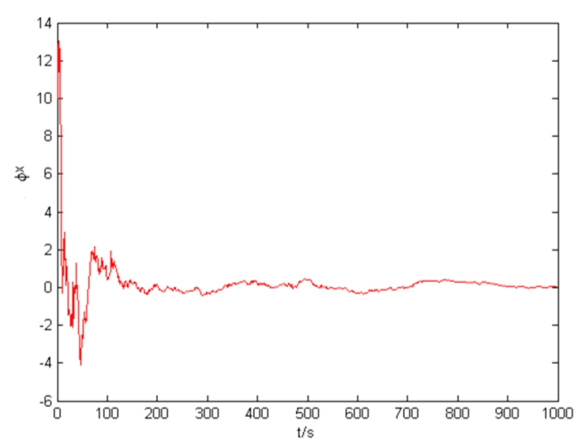

(a)

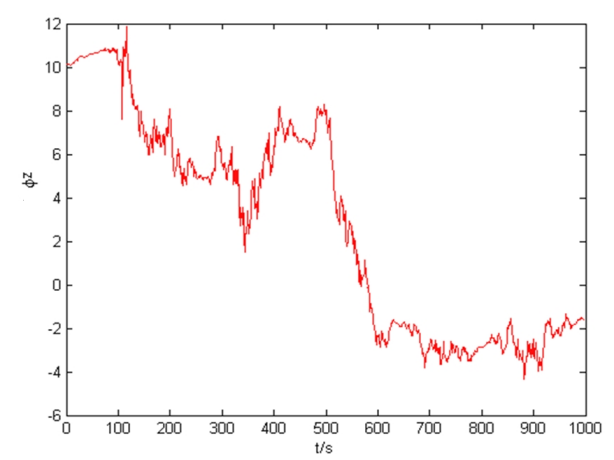

(c)

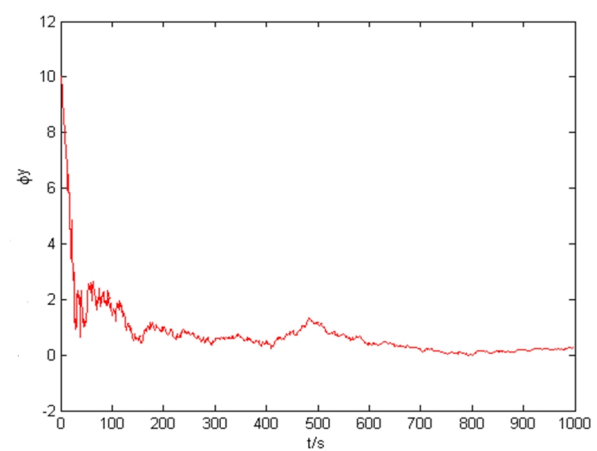

(b)

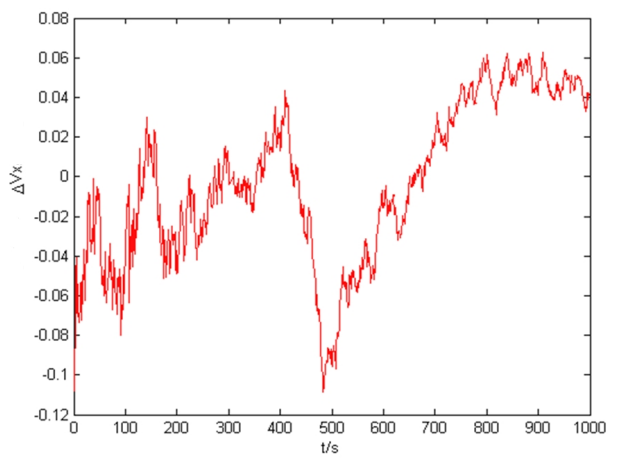

(d) 


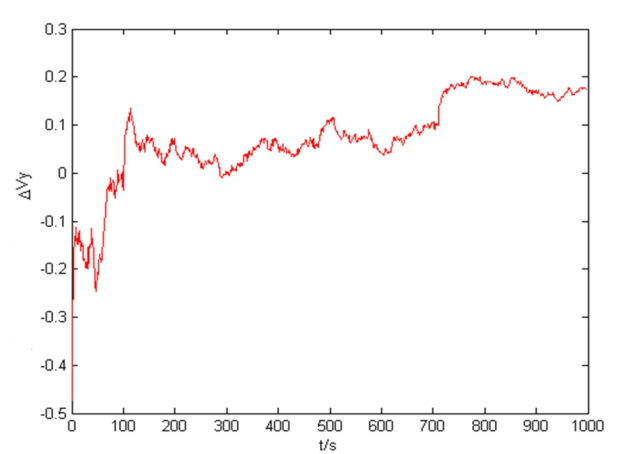

(e)

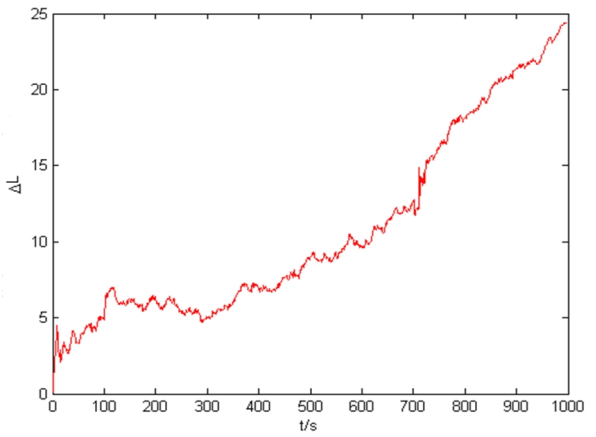

(f)

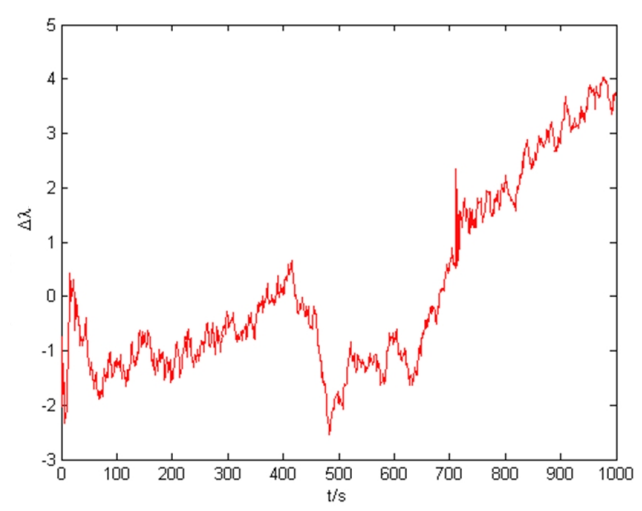

(g)

Fig. 2 Simulation results of hierarchical multi-level variable structure FDIR optimization detection

Fig. 2 shows that the Hierarchical multilevel variable structure FDIR has smaller global detection result estimation error, there basically no jump in curve, it shows that this method has higher accuracy and smooth reconstruction ability than the former.

\section{Summary}

Information distribution variables are collected through the sensor and detected into the system by using DBN inference, and the uncertainty of sensor measurement is integrated into the detection system in the DBN probabilistic network. The global optimization fusion method and the FDIR of the global system are realized, and the filtering estimation discontinuity which is caused by the frequent reset of the system is avoided, improve the accuracy and overall performance of the global fusion. The result of simulation shows that the proposed method is effective and reliable.

\section{References}

[1] Teng W.G. Information fusion for self-organizing sensor network. Emerging Information Technology Conference. 2005,1 3.

[2]Huang You-ping. Research on Bayesian network [D].Beijing: Chinese Academy of Science, 2005. [3] Sidar M.M, Doolin B.F. On the feasibility of real-time prediction of aircraft carrier motion at sea. IEEE Trans on Automatic Control, 1983,28(3), pp.350-355.

[4] KE Xizheng,XI Xiao-li,CHEN Jian.Concerning information configuration of integrated navigation system[J].Journal of Astronautics, 2004, 25(6),pp.632-636.

[5]Xu Ping, Wang Youcai, Navigation sensor fault detection method based on improved Kalman filter[A]; proceedings of the seventh Chinese information fusion conference[C].2015,pp.557-562.

[6] WANG Yu-fei, Huang Xian-lin, Hu Heng-zhang. Eigenvalue-decomposition-based adaptive information fusion filter algorithm for integrated navigation systems [J]. Acta Aeronautica et Astronautica Sinica, 2000,21(3),pp.274-276.

[7] Mu Rongjun,Cui Naigang. Theoretical and simulation research on optimizing information fusion method for multi-sensor[j].Chinese Journal of Scientific Instrument,2006,27(6),pp.326-328. 